\title{
Pseudoelasticity of NiTi Orthodontic Wires Modified by Current Methodologies: A Critical Comparison
}

\author{
G. Airoldi and G. Riva* \\ Dipartimento di Fisica, Universita' di Milano, via Celoria 16, 20133 Milano, Italy \\ * INFM, Unita di Milano Universita', via Celoria 16, 20133 Milano, Italy
}

\begin{abstract}
It is well known that applications of NiTi alloys in orthodontics rely on the pseudoelastic properties developed at temperatures higher than $\mathrm{A}_{\mathrm{f}}$. That implies as a necessary condition that the body temperature $\left(T_{b}\right)$ be higher than $A_{f}$; the level of reversion stress, $\sigma_{r}$ depends moreover upon the difference between $\mathrm{T}_{\mathrm{b}}$ and $\mathrm{A}_{\mathrm{f}}$. Nowadays the interest is directed to obtain an archwire with distinct $\sigma_{\mathrm{r}}$ in different segments: that means to modify locally the properties of the wire by local thermal treatments. Current methodologies exploit either the joule effect or a hot air thermal treatment: both have been here used on NiTi archwires to modify $\left(\mathrm{T}_{\mathrm{b}}-\mathrm{A}_{\mathrm{f}}\right)$ and, as a consequence, the expected $\sigma_{\mathrm{r}}$. The properties detected along the archwires by means of Differential Scanning Calorimetry, have been found quite disuniform when the active procedure of the resistive heat treatment was used, whilst good uniformity has been found when adopting the passive hot air heat treatment method. At the light of the present results, the hot air treatment method, allowing a better local temperature control, appears more reliable than the resistive heat treatment method.
\end{abstract}

\section{INTRODUCTION}

In recent years, the use of NiTi alloys in medical applications is steadily growing, but it was since the early 70's that their use in orthodontics was proposed to provide the force to accomplish the dental movement [1-2]. In the first applications, the shape memory effect was exploited: the wire, at room temperature in martensitic (M) phase, was pseudoplastically deformed, attached to the teeth and heated up to a temperature a little bit higher that the body temperature $\mathrm{T}_{\mathrm{b}}$. The wire developed a reversion force due to the constrained recovery, exploited in teeth re-alignment. However, this application was not straightforward, as the constrained recovery force depends upon many parameter as, for example, the initial pseudoplastic deformation, and the compliance of the system wire-tooth-bone: the former is very difficult to evaluate in the complex shapes usually given to the wire by the practitioner; the latter deals with the change of the recovery force during dental movements in a biological environment which modifies during the therapy, as a consequence of tissues response to the applied force. A prediction of the force developped by the wire was intrinsically complicated.

During the 80 's, as pseudoelastic NiTi wires became available on the market, it appeared that transformation pseudoelasticity was more easy to handle in the orthodontic practice. The "Variable Load, Constant Force and Cross-section Orthodontics" [3-4] (VLCFCO) aimed to exploit the unique benefits provided by the pseudoelastic force, constant and light as an optimal biological force is. The exploitation of superelastic NiTi allows both to correct malocclusions by appropriate forces in short periods (weeks against months); and, last but non 
least, to save chair-time thanks to the wide working deflection range provided by pseudoelasticity which does not require the repeated loadings typical of standard stainless steel wires.

By the way, it was during the 80 s that the term superelasticity came into use in the market, referring to $\mathrm{NiTi}$ wires which, whatever deformed (obviuosly below the plastic deformation limit of martensite but, anyway, well above the limit of traditional materials), totally recovered the deformation. This definition was, in a certain sense, misleading: at $\mathrm{T}>\mathrm{A}_{f}$, a NiTi wire where the work hardening prevented a good (i.e. constant force) pseudoelastic transformation behaviour, originating the so-called "linear superelasticity" [5], was confused with a wire where the correct thermomechanical treatment allowed to exploit a true constant pseudoelastic force. The terms "old" and "new" generation were introduced to discriminate wires showing either linear or constant force superelastic behaviour: obviously, only the new generation wires fulfill the requirements of the VLCFCO.

In the VLCFCO, however, if the superelasticity was very appealing for the above mentioned features, on the other hand, it actually prevented a current step in the orthodontic practice: the shaping of the wire. As a matter of fact, the wire must have a shape which, at the end of the therapy, bring the teeth to the positions correct for that specific clinical case. Using a traditional material (i.e. stainless steel) this is obtained by plastic deformation of the archwire: however, in NiTi, plastic deformation degrades or suppresses the superelastic behaviour. Hence, the use of NiTi wire was confined to that initial part of the orthodontic therapy, where large dental movements were required, with final positions not really the exact ones: a further fine alignment by conventional wires was required. Anyway, for this application, sets of NiTi archwires, with fixed choices of curvature radius, are currently available in order to match as better as possible the specific clinical case. In the practice, for a fixed cross-section, the orthodontist has to buy several packaged Ni'Ti wires whilst, in the traditional approach, he uses a unique roll of stainless steel wire. Last but not least, three dimensional curvatures (i.e. the Spee curve) or, in general, formability, in the case of NiTi are not afforded.

At the light of the previous remarks, even if the orthodontic applications are a wide market for NiTi alloys, their use does not fulfill the canonical orthodontic approach.

In recent years, to overcome the drawback related to the three dimensional shaping, NiTi archwires already Spee-shaped appeared on the market: however, this increases the number of NiTi archwires athands; realizing that the formability was a "must", NiTi wires, pseudoelastic at $\mathrm{T}_{\mathrm{b}}$ and in martensitic phase at room temperature, were introduced: they can be shaped exploiting the pseudoplastic accomodation of strains due to martensite de-twinning. However, after recovering at $T_{b}>A_{f}$, the final shape is not the shape impressed at room temperature by the orthodontist but, again, that imprinted by the producer. It is possible this will induce confusion in the field, as occurred in the 80's in relation to "old" and "new" generation NiTi wires. The true solution of the above stated problems, connected with a wider use of superelastic $\mathrm{NiTi}$ wires in the orthodontic practice, was proposed by [6] in the late 80's: one single straight NiTi wire can be submitted to an appropriate heating treatment, as usually done in the memory imprinting process, directly by the orthodontist, in order to obtain any desired three dimensional shape.

As the thermal treatments in NiTi shape memory alloys modifies the transformation temperatures and, as a consequence, the superelastic behaviour at $\mathrm{Tb}$, the superelastic response can be programmed, even in specific portions of the archwire, by performing local thermal treatments: this approach, extension of the VLCFCO, has been defined "Shape\&Force user-Programmable Orthodontics" (SFPO). A short local thermal treatment, "ad hoc" performed by the practitioner, is used to modify both the shape and the superelastic force developed by the archwire. This has been proved on commercial archwires [7-8], under laboratory controlled conditions. However, the SFPO has to struggle with the limits of the commercial conditioning equipments, exploiting either the Direct Electric Resistance Heating Treatment (DERHT) method or the Direct Hot Air Heating Treatment (DHAHT) method. As a matter of fact, during the "shape \& force" conditioning procedure, care must be paid to the thermal treatment, which, if uncorrectly performed, could destroy the superelastic behaviour.

In order to assess the best suited conditioning device for the SFPO, and, as a consequence, in 
order to put the basis of a clinical protocol, a comparative study on "shape \& force userprogrammable NiTi archwires", Spee-shaped by either of the above mentioned conditioning equipments, is here presented and critically discussed.

\section{EXPERIMENTAL}

Superelastic NiTi orthodontic archwires, marketed as Neosentalloy F100, F200, F300 (provided by GAC International Inc.), with a rectangular section $0.46 \times 0.64 \mathrm{~mm}^{2}$ were examined. The number which distinguishes the wires is related to the superelastic force (in grams) developed in a three point bending experiment, with $14 \mathrm{~mm}$ span length between knives.

Differential scanning calorimetry (DSC) measurements were performed by a Perkin-Elmer DSC7 equipment, at a scanning rate of $10^{\circ} \mathrm{C} / \mathrm{min}$. The temperature calibration was carried out by means of the melting point of In $\left(156.6^{\circ} \mathrm{C}\right)$ and $\mathrm{Hg}\left(-38.9^{\circ} \mathrm{C}\right)$ : the accuracy and precision of the temperature determination were within $\pm 0.1^{\circ} \mathrm{C}$. The NiTi wires were investigated either in the As Received (AR) condition or Spee-shaped by means of either the DERHT or

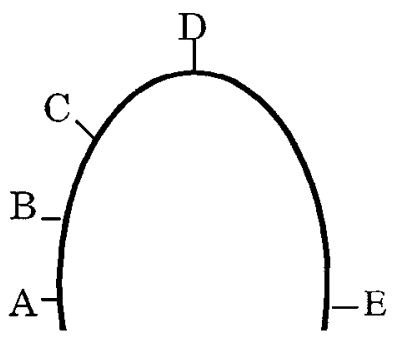

Fig.1: sampling positions of the investigated specimens along the NiTi archwire.

the DHAHT method. The heating treatments were performed by commercial equipments, the Archmate (by GAC International Inc., for DERHT) and the Weller AG 701S (by CooperTools, The Cooper Group Deutschland GmbH, D, for DHAHT). The DSC specimens were cut from the archwires in the positions labeled as schematically depicted in fig.1, to check the local properties of the heat treated wires.

\section{RESULTS}

In the $\mathrm{AR}$ condition, the archwires show a gray-silver surface color. After the spee-shaping, those heat treated by DHAHT keep their original surface colour while the ones treated by DERHT show an inhomogeneous coloration: from the original color in A, near the archholder, through a yellow/orange gradation $(A \rightarrow B \rightarrow C)$ with a local orange spot in $D$ where the stick was applied to give curvature.Fig.2 shows the DSC scans, on cooling, obtained on F100, F200 and F300 specimens in the AR condition. The two endothermic peaks are associated to the B2 $\rightarrow \mathrm{R}$ and $R \rightarrow M$ phase transition, well separated on the temperature scale. Fig. 3 shows the DSC scans on heating obtained on specimens sampled in positions A-D-E, for F100, F200, F300 archwires in the $A R$ condition. The exothermic peak is associated to the $M \rightarrow B 2$ phase transition. The homogeneity of the transformation behaviour along the archwire is clearly evident. Typical DSC scans of specimens in A-B-C-D positions, for F200 archwires, heat treated by either DERHT or DHAHT, are shown in figs.4-5 (on heating) and in figs.6-7 (on cooling). The $\mathrm{M} \rightarrow \mathrm{B} 2$ transformation temperatures (As, Apeak, Af) for F100, F200 and F300 specimens in the AR condition (sampling positions A-D-E) or Spee-shaped (sampling positions A-B-C-D), the latter obtained by either DERHT or DHAHT, are reported in Table I.

\section{DISCUSSION}

In the AR condition, the investigated F100, F200 and F300 show a good homogeneity of the thermodynamic properties along the archwire. As expected from the Clausius-Clapeyron-like law [9], the force developed at $T_{b}$ by the F100, F200, F300 archwires ranks with $\left(T_{b}-A_{f}\right)$. In the archwires Spee-shaped by DERHT, the inhomogeneous coloration, due to oxide layers different in thickness, suggests an heating treatment not uniform along the archwire. This is supported by the inhomogeneities in the thermodynamic properties, as shown in figs. 4 and 6 , 


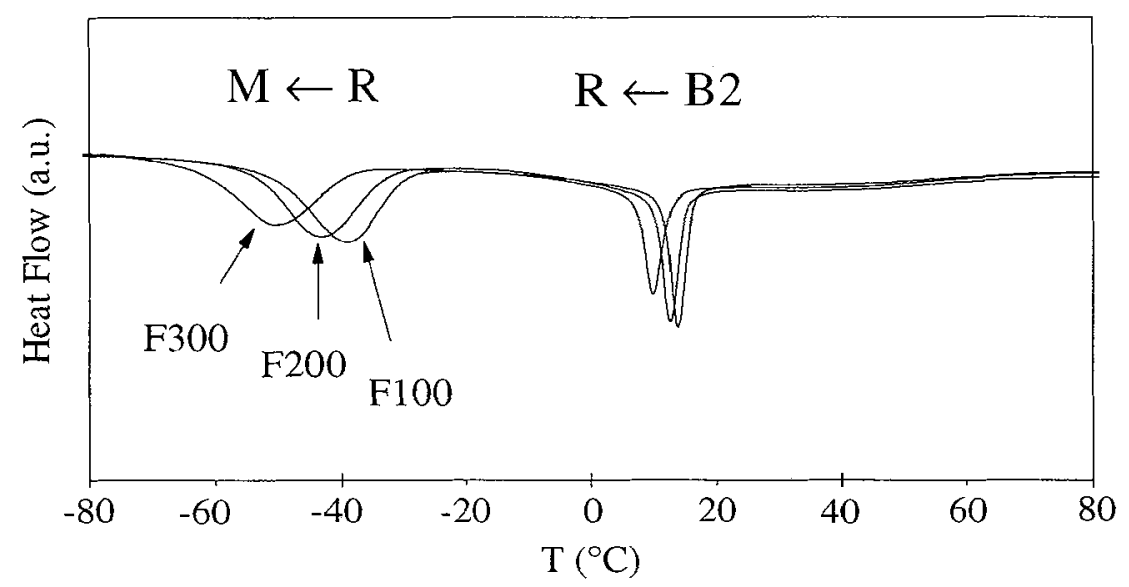

Fig.2: DSC scans on cooling obtained on F100, F200 and F300 specimens in the As Received condition.

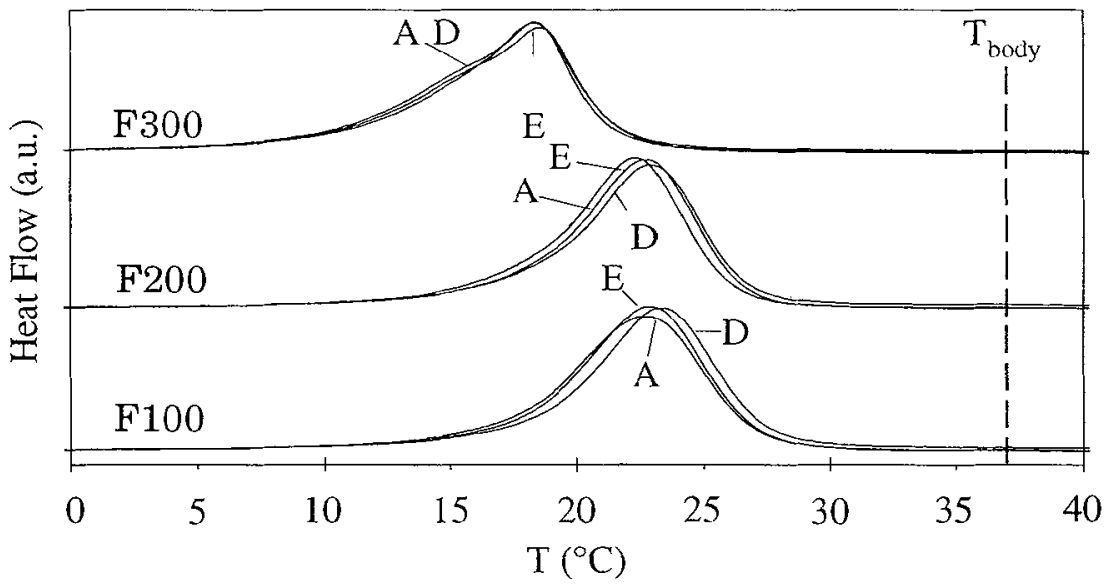

Fig.3: DSC scans obtained on specimens sampled in positions A-D-E (see fig. 1) of F100, F200, F300 archwires. The vertical dashed line refers to $\mathrm{T}_{\mathrm{body}}$.

where it appears that the transformation behaviour both in the position A (very near the massive grips) and in the $\mathrm{D}$ one (where the stick was applied to give curvature) is quite similar but strongly different from the B-C positions: this evidences the efficiency of the stick, and, in general of whatever is used to ply the wire, as heat sink. It has already been shown, under laboratory controlled conditions, that, on similar archwires [8], the thermal treatment progressively shifts towards high temperature the $\mathrm{M} \rightarrow \mathrm{B} 2$ transformation temperatures, as a function of both the temperature $T$ and the treatment time $t$ : that implies that, when $\mathrm{T}_{b}<\mathrm{A}_{f}$, the superelastic behaviour is lost at $\mathrm{T}_{\mathrm{b}}$. As $t$, the time the electric current flows, is preselected in the DERHT, it can be deduced from experimental data that a non uniform temperature sets in during treatment and, as a consequence, a superelastic behaviour, modified in an unpredicatble way, is expected along the archwire. At the light of the above results, it can be concluded that in the DERHT method it looks difficult to control the temperature in a predictable way along the archwire.

On the contrary, in the DHAHT method, the temperature is preselected. Actually a thermal gradient exists in the direction of the exhausted air flow [10] but the maximum temperature is fixed: no matter how long the treatment time is, an overheating cannot occur. Once chosen the appropriate treatment 


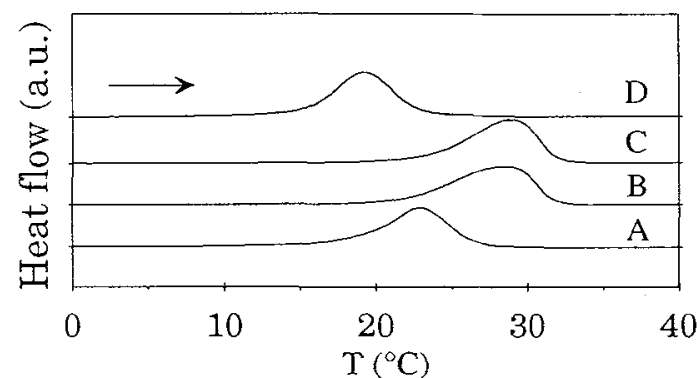

Fig.4: DSC scans, on heating, of A-B-C-D specimen of a F200 archwire, Spee-shaped by DERHT method.

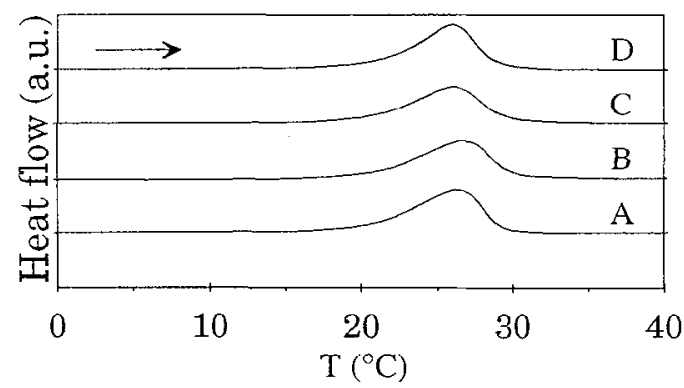

Fig.5: DSC scans, on heating, of A-B-C-D specimen of a F200 archwire, Spee-shaped by DHAHT method.

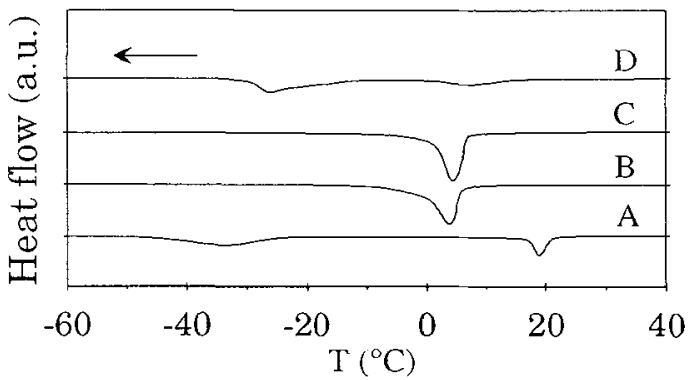

Fig.6: DSC scans, on cooling, of A-B-C-D specimen of a F200 archwire, Spee-shaped by DERHT method.

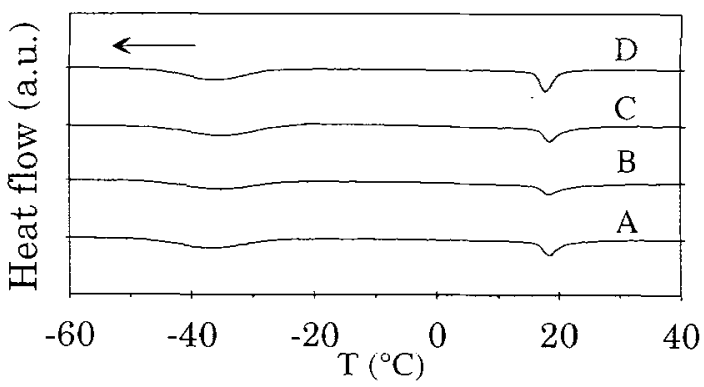

Fig.7: DSC scans, on cooling, of A-B-C-D specimen of a F200 archwire, Spee-shaped by DHAHT method.

\section{Table I}

$\mathrm{M} \rightarrow \mathrm{B} 2$ transformation temperatures (in ${ }^{\circ} \mathrm{C}$ ) for F100, F200 and F300 archwires, in the sampling positions shown in fig1., in the As Received condition or Spee-shaped by either DERHT or DHAHT.

F100

\section{Sampling position}

$\begin{array}{llllll}A R & A & B & C & D & E\end{array}$

$\mathrm{A}_{s} \quad 18.2=\quad=\quad 19.017 .9$

$\mathrm{A}_{\text {peak }} 23.9=\quad=24.323 .6$

$\mathrm{A}_{\mathrm{f}} \quad 28.1=28.528 .0$

$\begin{array}{llllll}\text { DHAHT A } & \text { B } & \text { C } & \text { D } & \text { E }\end{array}$

$\begin{array}{lllll}\mathrm{A}_{s} & 17.3 & 16.2 & 17.5 & 17.4=\end{array}$

$\begin{array}{lllll}\mathrm{A}_{\text {peak }} & 24.1 & 23.8 & 24.5 & 24.6\end{array}$

$\begin{array}{lllll}\mathrm{A}_{\mathrm{f}} & 29.2 & 29.9 & 30.1 & 29.8=\end{array}$

$\begin{array}{llllll}\text { DERHT A } & \text { B } & \text { C } & \text { D } & \mathbf{E}\end{array}$

$\begin{array}{lllll}\text { As } & 21.1 & 20.1 & 18.0 & 12.2=\end{array}=$

$\begin{array}{lllll}\mathrm{A}_{\text {peak }} & 23.2 & 27.7 & 25.0 & 18.9=\end{array}$

$\begin{array}{lllll}\mathrm{A}_{\mathrm{f}} & 27.5 & 33.6 & 31.5 & 24.6=\end{array}$

\section{F200 Sampling position}

$\begin{array}{llllll}A R & A & B & C & D & E\end{array}$

As $\quad 18.6=\quad=\quad 19.018 .4$

$\mathrm{A}_{\text {peak }} 21.6=23.923 .3$

$\mathrm{A}_{\mathrm{f}} \quad 27.6=27.727 .1$

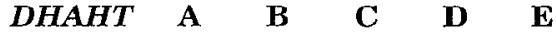

$\begin{array}{lllll}\text { A } & 21.6 & 21.2 & 21.0 & 20.5\end{array}=$

$\begin{array}{lllll}A_{\text {peak }} & 25.9 & 25.9 & 26.6 & 26.3=\end{array}$

$\begin{array}{lllll}\mathrm{A}_{\mathrm{f}} & 28.8 & 29.4 & 29.9 & 29.2\end{array}$

$\begin{array}{llllll}\text { DERHT A } & \text { B } & \text { C } & \text { D } & \text { E }\end{array}$

$\begin{array}{lllll}\text { A.s } & 18.7 & 21.8 & 23.5 & 15.3\end{array}=$

$\begin{array}{lllll}\text { Apeak } & 23.0 & 28.4 & 28.9 & 19.3=\end{array}$

$\begin{array}{lllll}\text { Af } & 26.5 & 31.8 & 32.0 & 22.7\end{array}=$

F300 Sampling position

$\begin{array}{llllll}A R & \text { A } & \text { B } & \text { C } & \text { D } & \text { E }\end{array}$

$\mathrm{A}_{\mathrm{s}} \quad 13.2=\quad=\quad 13.713 .3$

$A_{\text {peak }} 19.2=\quad=\quad 19.119 .4$

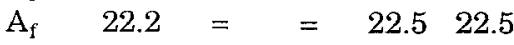

$\begin{array}{llllll}\text { DHAHT } & \text { A } & \text { B } & \text { C } & \text { D } & \text { E }\end{array}$

$\begin{array}{lllll}\mathrm{A}_{4} & 11.2 & 11.0 & 12.7 & 14.2=\end{array}$

$\begin{array}{llllll}\mathrm{A}_{\text {peak }} & 18.4 & 18.4 & 18.5 & 18.6=\end{array}$

$\begin{array}{lllll}\mathrm{A}_{\mathrm{f}} & 21.3 & 21.8 & 21.3 & 20.8\end{array}$

$\begin{array}{llllll}\text { DERHT } & \text { A } & \text { B } & \text { C } & \text { D } & \text { E }\end{array}$

$\begin{array}{lllll}\text { As } & 14.6 & 24.8 & 24.6 & 16.7=\end{array}$

$\begin{array}{lllll}A_{\text {peak }} & 18.6 & 29.8 & 29.2 & 20.8=\end{array}$

$\begin{array}{lllll}A_{\mathrm{f}} & 20.8 & 32.5 & 32.3 & 30.6\end{array}=$ 
temperature, only time remains as a free parameter, allowing a good control of the overall "shaping \& programming" process. As a matter of fact a good homogeneity is found both in the transformation behaviour (figs.5,7) and in the transformation temperatures. After the present heat treatment, for F100 and F200 specimens, the transformation temperatures are a little bit higher in comparison with the ones in the $A R$ condition, in agreement with the results already found [8]: a lower superelastic force at $T_{b}$ is expected. The results found for F300 specimens, however, do not agree with previous ones [7-8], being the transformation temperatures a little bit lower. A temptative hypothesis, even if the composition and the thermomechanical history of the wires is not known, could be the following. It is well known that cold drawn wires [11], submitted to aging treatments in the $300^{\circ} \mathrm{C}-600^{\circ} \mathrm{C}$, undergo several physical metallurgical processes (e.g. recovery, precipitation , recrystallization) which modify the thermoelastic martensitic transformation, by altering both the chemical and the non-chemical contributions to the thermoelastic balance. Moreover, it has been observed [12] that, in a cold drawn Ni-rich alloy, as a consequence of ageing, the transformation temperatures decrease in the first time stage and afterwards steadily increase and saturate. Thus, assuming that the alloy used for F100, F200 and F300 is the same, and that the control of superelastic behaviour at $\mathrm{T}_{\mathrm{b}}$ is accomplished by a proper thermal treatment during the archwire shaping, the F300 in the AR condition could be still in the first stage and hence, the short thermal treatment here performed could lower the transformation temperatures. In fact, under laboratory controlled conditions, for similar F300 specimen, the transformation temperatures were observed to increase for long treatment times (up to $3.6 \mathrm{ks}$ ).

\section{CONCLUSION}

With the aim to develop a clinical protocol for the Shape\&Force user-Programmable Orthodontics, NiTi superelastic wires shaped by the two currently used methodologies, have been investigated. It has been proved that the hot air method (DHAHT) is more reliable than the one exploiting the resistive heating, which guarantees neither a good temperature control nor temperature uniformity along the archwire.

\section{ACKNOWLEDGEMENTS}

The support of MUSRT 40\% and of 93.01538.CT11 CNR are gratefully acknowledged. Thanks are due to Dr.A.Radicchi (Clinica Odontoiatrica, Università di Roma I), for the preparation of the Spee-shaped archwires by DERHT and DHAHT.

\section{REFERENCES}

[1] Andreasen G. and Brady P., The Angle Orth. 42 (1972) 172-177

[2] Andreasen G., Am.J.Orthod. 78 (1980) 528-537

[3] Miura F., Mogi M., Ohura Y., Hamanaka H., Am.J.Orthodon. 90 (1986) 1-10

[4] Sachdeva R.,Fukuyo S.,Suzuki K.,Oshida Y. and Miyazaki S.,Mat.Sc.Forum 56(1990)693-698

[5] Zadno G. and Duerig'T., "Linear and non-linear superelasticity in NiTI",MRS Int'l Mtg. on Adv. Materials, may 30 - june 3 1988, Tokyo, (J) (Materials Research Society, 1989), pp.201-206

[6] Miura F, Mogi M. and Ohura Y., Eur.J.Orthod. 10 (1988) 187-191

[7] Airoldi G. and Riva G., "Pre-programmed shape memory NiTi orthodontic wires", in Topics in Biomedical Physics, eds. L.Andreucci and A.Schenone, (World Scientific, Singapore, 1992) pp.486-491

[8] Airoldi G. and Riva G., Phys.Med. IX Suppl.1 (1993) 81-83

[9] Wollants P., DeBonte M. and Roos J.R., Z.Metallkde $10 \quad$ (1979) 113-117

[10] Airoldi G., Riva G. and Vanelli M., unpublished results.

[11] Todoroki T. and Tamura H., Trans.Jpn.Inst.Met. 28 (1987) 83-94

[12] Funakubo H., Shape Memory Alloys (Gordon \& Breach Science Publisher, 1987), pp.110-113 